\title{
artigo
}

\section{Eficácia da clorexidina no contexto da covid-19: revisão integrativa}

\author{
Effectiveness of chlorexidine in the context of covid-19: integrative review
}

Eficacia de la clorexidina en el contexto del covid-19: revisión integrativa

\begin{abstract}
RESUMO
Objetivo: analisar a eficácia da clorexidina sobre a infecção do COVID-19. Métodos: Realizou-se uma revisão integrativa da literatura, a coleta de dados foi realizada entre a segunda e terceira semana de junho de 2021, tendo por limite temporal estudos publicados entre março de 2020 e junho de 2021, nas bases de dados PubMed, Lillacs e Clinical Trials. Resultados: A pesquisa identificou 70 artigos publicados no período de eleição, destes, 35 foram incluídos na revisão, sendo que 6 não tratavam da temática, e dos 27 restantes, 15 indicavam que a clorexidina tem um resultado positivo sobre o SARS-Cov-2, 6 indicaram que ela é ineficaz e 6 obtiveram resultados inconclusivos. Conclusão: Apesar da maioria dos estudos revisados indicarem pelo uso da clorexidina com eficácia sobre a COVID-19, o pequeno número de pesquisas sobre o tema e a falta de resultados robustos, indicam a necessidade de mais pesquisas sobre o efeito da substância no combate a infecção.
\end{abstract}

DESCRITORES: COVID-19; CLOREXIDINA; SARS-COV-2.

\section{ABSTRACT}

Objective: to analyze the effectiveness of chlorhexidine on COVID-19 infection. Methods: An integrative literature review was carried out, data collection was carried out between the second and third week of June 2021, having as a time limit studies published between March 2020 and June 2021, in the PubMed databases, Lillacs and Clinical Trials. Results: The search identified 70 articles published in the election period, of which 35 were included in the review, 6 of which did not address the topic, and of the remaining 27, 15 indicated that chlorhexidine has a positive result on SARS-Cov-2, 6 indicated that it is ineffective and 6 obtained inconclusive results. Conclusion: Although most of the reviewed studies indicate the use of chlorhexidine effectively against COVID-19, the small number of researches on the subject and the lack of robust results indicate the need for more research on the effect of the substance in combating infection.

DESCRIPTORS: COVID-19; Chlorhexidine; SARS-Cov-2.

\section{RESUMEN}

Objetivo: analizar la efectividad de la clorhexidina sobre la infección por COVID-19. Métodos: Se realizó una revisión integradora de la literatura, la recolección de datos se realizó entre la segunda y tercera semana de junio de 2021, teniendo como límite de tiempo los estudios publicados entre marzo de 2020 y junio de 2021, en las bases de datos PubMed, Lillacs y Clinical Trials. Resultados: La búsqueda identificó 70 artículos publicados en el período electoral, de los cuales 35 fueron incluidos en la revisión, 6 de los cuales no abordaron el tema, y de los 27 restantes, 15 indicaron que la clorhexidina tiene resultado positivo en SARS-Cov- 2, 6 indicaron que es ineficaz y 6 obtuvieron resultados no concluyentes. Conclusión: Aunque la mayoría de los estudios revisados indican el uso de clorhexidina de manera efectiva contra COVID-19, el pequeño número de investigaciones sobre el tema y la falta de resultados sólidos indican la necesidad de más investigación sobre el efecto de la sustancia en la lucha contra la infección.

DESCRIPTORES: COVID-19; Clorhexidina; SARS-Cov-2.

\section{Paola e Silva Nunes}

Odontóloga. Universidad de Ciencias Empresariales y Sociales - UCES

ORCID: 0000-0001-6789-741 
CARLO ENDRIGO BUENO NUNES

Médico. Universidad de Ciencias Empresariales y Sociales - UCES

ORCID: 0000-0002-6308-7284

\section{INTRODUÇÃO}

O ano de 2020 foi marcado pelo surgimento da pandemia do Novo Coronavírus, decretada em 11 de março de 2020 pela Organização Mundial de Saúde (OMS), sendo que, nesta data, ela já estava presente em aproximadamente 114 países e atingido mais de 118 mil pessoas com sintomas de SARS-Cov- ${ }^{1}$.

O SARS-CoV-2, um acrônimo, em inglês, de "síndrome respiratório agudo severo causado por coronavírus 2 "2 é um vírus da família do coronavírus e foi identificado na China, no final de 2019 e por isso recebeu o nome de COVID-19, pertencendo a uma grande família de vírus que afeta o sistema respiratório.

O SARS-CoV-2 já foi encontrado em vários tecidos do corpo humano, mas é nas células do pulmão (pneumócitos) e do intestino (enterócitos) que se encontra a maior quantidade de vírus. A possibilidade de transmissão do vírus por aerossóis, tornou-se mais robusta após a identificação de que enzimas conversoras de angiotensina 2 (ACE2), um receptor de superfície celular necessário para a entrada do vírus na célula humana, também está presente nas células da mucosa oral e que o vírus é encontrado na saliva, apesar de apresentar maior carga viral na orofaringe ${ }^{3}$.

Infecções virais são transmitidas principalmente por contato com superfícies ambientais contaminadas e por aerossolização ${ }^{4}$. Patógenos virais podem sobreviver em superfícies ambientais por vários dias, no caso da COVID-19, pode sobreviver por pelo menos 3 dias em vários materiais presentes em salas de cirurgia, como aço inoxidável e plástico ${ }^{5}$.

Isso torna a necessidade e importância da desinfecção do ambiente ainda mais importante, Há muito tempo se tem usado a nível hospitalar e ambulatorial uma biguana com propriedades hidrofílicas e hidrofóbicas que é a Clorexidina. A clorexidina é
O SARS-CoV-2 já foi encontrado em vários tecidos do corpo humano, mas é nas células do pulmão (pneumócitos) e do intestino (enterócitos) que

se encontra a maior quantidade de vírus uma substância com ação antimicrobiana, eficaz no controle da proliferação de bactérias na pele e mucosas, sendo um produto muito utilizado como antisséptico na prevenção de infecções ${ }^{6}$.

Foi introduzida no mercado em 1954 como antisséptico para feridas cutâneas e foi comercializada nas formas de acetato, cloridrato e digluconato. Logo se destacou pelas características bactericidas altas, pela baixa toxidade e alta substantividade ${ }^{7}$. Em altas concentrações é bactericida e em baixas concentrações tem efeito bacteriostático, mantendo-se nos tecidos por período de $12 \mathrm{~h}$.

Além disso, não causa resistência microbiológica, não é corrosivo, não apresenta odor e não é contaminante ${ }^{8}$, o que fez a clorexidina tornar-se o padrão-ouro em comparação com outros agentes antimicrobianos ${ }^{9}$.

Há uma necessidade imensa de aumentar a conscientização entre os profissionais sobre a atividade viricida de enxaguatórios bucais disponíveis no mercado, conforme demonstrado por vários estudos in vitro, $\mathrm{e}$ exortar os profissionais de saúde a realizar mais ensaios clínicos e dar um passo de translação em direção à prática clínica ${ }^{10}$.

No contexto da pandemia do COVID-19, vários estudos buscaram entender a eficácia da clorexidina na infecção do SARS-CoV-2, já que pouco se sabia sobre este vírus e quais produtos poderiam ter ação sobre ele. Alguns dos desafios da COVID-19 incluem a qualidade das informações sobre a doença, as adaptações relativas à biossegurança, geração de protocolos, o medo e a ansiedade presentes no cotidiano dos profissionais no atual contexto sanitário ${ }^{11}$.

Buscando obter evidências sobre a ação que a clorexidina pode ter sobre o Novo Coronavírus, realizou-se uma revisão integrativa, que é um método que proporciona a síntese de conhecimento e a incorporação da aplicabilidade de resultados de estudos 


\section{artigo}

significativos na prática ${ }^{12}$, com o objetivo de sintetizar os estudos e analisar a eficácia da clorexidina sobre a infecção do COVID-19 e responder a seguinte pergunta: a clorexidina é eficaz como agente antimicrobiano na infecção pela COVID-19?

\section{MÉTODOS}

Trata-se de um estudo com coleta de dados realizada a partir de fontes secundárias, por meio de levantamento bibliográfico para a realização de uma revisão integrativa. Foram seguidos os seguintes passos: $1^{\circ}$ ) identificação do problema (definindo o propósito da revisão); $2^{\circ}$ ) a busca na literatura (com delimitação das palavras-chave, bases de dados e aplicação dos critérios definidos para a seleção dos artigos); $3^{\circ}$ ) a coleta de dados, utilizando o instrumento de coleta de dados previamente validado; $4^{\circ}$ ) a análise crítica dos estudos incluídos; $5^{\circ}$ ) a discussão dos resultados e $6^{\circ}$ ) a apresentação da revisão integrativa.

A busca dos estudos foi realizada nas plataformas: Literatura Latino-Americana e do Caribe em Ciências da Saúde (LILACS), PUBMED, que é um serviço da U. S. National Library of Medicine (NLM) e Clinical Trials, que é um registro nacional da web de estudos de pesquisa com apoio federal e privado, conduzidos nos Estados Unidos e em todo o mundo, onde encontramos estudos em diferentes status, desde o recrutamento até concluídos ${ }^{13}$. Está busca foi irrealizada no período entre a segunda e terceira semana de junho de 2021, seguindo os seguintes critérios de inclusão: artigos em inglês, espanhol e português, publicados entre março de 2020 e junho de 2021, cuja temática fosse a utilização da clorexidina para reduzir a contaminação pela infecção do SARS-CoV-2 (COVID-19).

Para a realização da busca, foram utilizadas combinações entre os seguintes Descritores em Ciências de Saúde (DeCS) e Medical Subject Headings ( $\mathrm{MeSH}$ ), utilizando-se os operadores boleanos OR entre termos semelhantes e AND entre os descritores, como visto no quadro 1 .

Nesta busca, foram identificados 70 artigos, sendo 22 estudos registrados no Cli-

\section{QUADRO 1: CONSTRUÇÃO DO DECS E MESH}

(("clorexidina" [Termos MeSH] OR "clorexidina" [Todos os Campos] OR "cloresidina" [Todos os Campos]) AND ("covid 19" [Todos os Campos] OR "covid 19" [Termos MeSH] OR "vacinas covid 19 "[Todos os campos] OR" vacinas covid 19 "[Termos MeSH] OR" seroterapia covid 19 "[Todos os campos] OR" seroterapia covid 19 "[Conceito suplementar] OR" teste de ácido nucleico covid 19 "[Todos os campos] OR" covid Teste de ácido nucleico 19 "[Termos do MeSH] OR" teste sorológico covid 19 "[Todos os campos] OR" teste sorológico covid 19 "[Termos do MeSH] OR" teste 19 covid "[Todos os campos] OR" teste 19 covid "[Termos do MeSH] OR "sars cov 2" [Todos os campos] OR "sars cov 2" [Termos MeSH] OR "sindrome respiratória aguda grave coronavírus 2"[Todos os campos] OR "ncov" [Todos os campos] OR "2019 ncov" [Todos os campos] OR (("coronavirus" [Termos do MeSH] OR "coronavirus" [Todos os campos] OR "cov" [Todos os campos]) AND 2019 / 11/01: 3000/12/31 [Data - Publicaçāo]))) E (2020: 2021 [pdat]).

Fonte: Nunes, PS; Nunes, V.2021

\section{FIGURA 1- FLUXOGRAMA DA ANÁLISE DAS BASES DE DADOS}

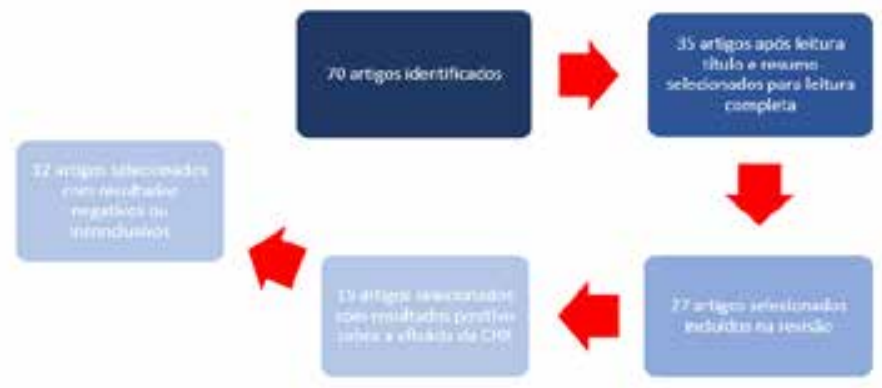

Fonte: Nunes, PS; Nunes, CEB. 2021

nical Trials, 1 estudo na base LILACS e 47 na base PUBMED, sendo que todos foram transferidos para o instrumento de coleta de dados validado (14) e 2 pesquisadores independentes e blindados, fizeram a leitura inicial dos títulos e resumos, resultando na inclusão de 35 estudos que contemplavam os critérios de inclusão e que foram lidos por completo, como demonstrado no fluxograma da figura 1 .

\section{RESULTADOS}

Após a leitura pelos avaliadores independentes dos títulos e resumos dos 70 artigos obtidos com a pesquisa nas bases de dados, 13 (LILACS e PUBMED) foram excluídos por não tratarem da temática da revisão. Dos 22 registros no ClinicalTrials. gov, apenas 3 estavam concluídos, porém, 1 não fazia parte da temática da revisão e nenhum apresentava os resultados. Os 35 restantes, foram lidos integralmente e destes 27 foram incluídos na revisão, sendo que 15 encontraram resultados positivos para a eficácia da clorexidina e 12 encontraram resultados ineficazes ou inconclusivos, como demonstra o quadro 2 .

\section{DISCUSSÃO}

A pandemia de COVID-19 instalada 


\begin{tabular}{|c|c|c|}
\hline \multicolumn{3}{|c|}{$\begin{array}{l}\text { QUADRO 1- Apresentação das publicações científicas referentes aos cuidados } \\
\text { de enfermagem para às pessoas com doenças crônicas acometidas por COVID-19: }\end{array}$} \\
\hline ESTUDO E ANO & AUTOR & EFICÁCIA \\
\hline Use of mouthwashes against COVID-19 in & Vergara-Buenaventura A,Castro-Ruiz C & Positiva \\
\hline Dentistry. (2020) & Moosavi MS, Aminishakib P, Ansari M & Positiva \\
\hline $\begin{array}{l}\text { Antiviral mouthwashes: possible benefit } \\
\text { for COVID-19 with evidence-based approach. (2020) }\end{array}$ & $\begin{array}{l}\text { Burton MJ, Clarkson JE, Goulao B,Glenny } \\
\text { AM, McBain AJ, Schilder AG, Webster KE, } \\
\text { Worthington HV }\end{array}$ & Inconclusivo \\
\hline $\begin{array}{l}\text { Antimicrobial mouthwashes (gargling) and nasal } \\
\text { sprays administered to patients with suspected or } \\
\text { confirmed COVID-19 infection to } \\
\text { improve patient outcomes and to protect healthcare } \\
\text { workers treating them. (2020) }\end{array}$ & Kampf G, Todt D, Pfaender S, Steinmann E & Inconclusivo \\
\hline $\begin{array}{c}\text { Persistence of coronaviruses on } \\
\text { inanimate surfaces and their inactivation with } \\
\text { biocidal agents. (2020) }\end{array}$ & $\begin{array}{l}\text { Yoon JG, Yoon J, Song JY, Yoon SY, Lim CS, } \\
\text { Seong H, Noh JY, Cheong HJ, Kim WJ. }\end{array}$ & Positiva \\
\hline $\begin{array}{l}\text { Clinical Significance of a High SARS-CoV-2 Viral Load } \\
\text { in the Saliva. (2020) }\end{array}$ & $\begin{array}{c}\text { Carrouel F, Gonçalves LS, Conte MP, Campus } \\
\text { G, Fisher J, Fraticelli L, Gadea- Deschamps E, } \\
\text { Ottolenghi L, Bourgeois D }\end{array}$ & Positiva \\
\hline $\begin{array}{l}\text { Antiviral Activity of Reagents in Mouth } \\
\text { Rinses against SARS-CoV-2. (2020) }\end{array}$ & $\begin{array}{l}\text { Seneviratne CJ, Balan P, Ko KKK, Udawatte } \\
\text { NS, Lai D, Ng DHL, Venkatachalam I, Lim KS, } \\
\text { Ling ML, Oon L, Goh BT, Sim XY] }\end{array}$ & Positiva \\
\hline $\begin{array}{c}\text { Efficacy of commercial mouth-rinses on } \\
\text { SARS-CoV-2 viral load in saliva: randomized control } \\
\text { trial in Singapore. } \\
\text { Infection. (2021) }\end{array}$ & León Molina J, Abad-Corpa E & Negativa \\
\hline $\begin{array}{l}\text { Disinfectants and antiseptics facing } \\
\text { coronavirus: synthesis of evidence and } \\
\text { recommendations. (2021) }\end{array}$ & Khokhar M, Roy D, Purohit P, Goyal M, Setia P & Positiva \\
\hline $\begin{array}{l}\text { Viricidal treatments for } \\
\text { prevention of coronavirus infection. (2020) } \\
\text { A critical appraisal of evidence in } \\
\text { the use of preprocedural mouthwash to avoid SARS- } \\
\text { CoV-2 transmission during oral } \\
\text { interventions. (2020) }\end{array}$ & $\begin{array}{l}\text { Sette-de-Souza PH, Soares Martins JC, } \\
\text { Martins-de-Barros AV, Rodrigues Vieira B, } \\
\text { Fernandes Costa MJ, da Costa Araújo FA }\end{array}$ & Inconclusivo \\
\hline $\begin{array}{l}\text { COVID-19 and Oral Surgery: A narrative review of } \\
\text { preoperative mouth } \\
\text { Rinses. (2020) }\end{array}$ & $\begin{array}{l}\text { Testori T, Wang HL, Basso M, Bordini G, Dian } \\
\text { A, Vitelli C, Miletic I, Del Fabbro M }\end{array}$ & Positiva \\
\hline $\begin{array}{l}\text { Interventions to reduce contaminated aerosols } \\
\text { produced during dental } \\
\text { procedures for preventing infectious diseases. (2020) }\end{array}$ & $\begin{array}{l}\text { Kumbargere Nagraj S, Eachempati P, Paisi M, } \\
\text { Nasser M, Sivaramakrishnan G, Verbeek JH }\end{array}$ & Inconclusivo \\
\hline
\end{tabular}




\section{artigo}

Nunes, P. S., Nunes, C. E. B.

Eficácia da clorexidina no contexto da covid-19: revisão integrativa

\begin{tabular}{|c|c|c|}
\hline $\begin{array}{c}\text { Interventions to Reduce Aerosolized } \\
\text { Microbes in Dental Practice: A Systematic Review } \\
\text { with Network Meta-analysis of } \\
\text { Randomized Controlled Trials. (2020) }\end{array}$ & Koletsi D, Belibasakis GN, Eliades T & Positiva \\
\hline $\begin{array}{l}\text { Could antiseptic } \\
\text { gargling prevent COVID-19? (2020) }\end{array}$ & $\begin{array}{l}\text { Mohamed NA, Ahmad Zainol Hady A, Abdul } \\
\text { Aziz AH, Isahak I }\end{array}$ & Inconclusivo \\
\hline $\begin{array}{l}\text { Safety alert for hospital environments and } \\
\text { health professional: chlorhexidine is ineffective for } \\
\text { coronavirus. (2020) }\end{array}$ & Assis MS, Araújo RAAM, Lopes AMM & Negativa \\
\hline $\begin{array}{l}\text { Potential Role } \\
\text { of Oral Rinses Targeting the Viral Lipid Envelope in } \\
\text { SARS-CoV-2 Infection. (2020) }\end{array}$ & $\begin{array}{l}\text { O'Donnell VB, Thomas D, Stanton R, Maillard } \\
\text { JY, Murphy RC, Jones SA, } \\
\text { Humphreys I, Wakelam MJO, Fegan C, Wise } \\
\text { MP, Bosch A, Sattar AS }\end{array}$ & Inconclusivo \\
\hline $\begin{array}{c}\text { Differential effects of antiseptic mouth rinses on } \\
\text { SARS-CoV-2 infectivity in } \\
\text { Vitro. (2021) }\end{array}$ & $\begin{array}{c}\text { Xu C, Wang A, Hoskin ER, Cugini C, Markowitz } \\
\text { K, Chang TL, Fine DH }\end{array}$ & Positiva \\
\hline $\begin{array}{l}\text { Physicochemical susceptibility of SARS-CoV-2 } \\
\text { to disinfection and physical approach of prophylaxis. } \\
\text { (2020) }\end{array}$ & Saadatpour F, Mohammadipanah F & Negativa \\
\hline $\begin{array}{l}\text { Use of chlorhexidine to eradicate oropharyngeal } \\
\text { SARS- } \\
\text { CoV-2 in COVID-19 patients. (2021) }\end{array}$ & Huang YH, Huang JT & Positiva \\
\hline $\begin{array}{l}\text { Dental workers in front-line of COVID-19: an in silico } \\
\text { evaluation } \\
\text { targeting their prevention. (2021) }\end{array}$ & $\begin{array}{c}\text { Sette-DE-Souza PH, Costa MJF, Amaral- } \\
\text { Machado L, Araújo FADC, Almeida Filho AT, } \\
\text { Lima LRA }\end{array}$ & Positiva \\
\hline $\begin{array}{l}\text { Methods to disinfect and decontaminate SARS- } \\
\text { CoV-2: a systematic } \\
\text { review of in vitro studies. (2021) }\end{array}$ & $\begin{array}{c}\text { Kwok CS, Dashti M, Tafuro J, Nasiri M, } \\
\text { Muntean EA, Wong N, Kemp T, Hills G, Mallen } \\
\text { CD }\end{array}$ & Negativa \\
\hline $\begin{array}{l}\text { Chlorhexidine: An effective anticovid mouth rinse. } \\
\qquad \text { (2021) }\end{array}$ & $\begin{array}{c}\text { KJain A, Grover V, Singh C, Sharma A, Das DK, } \\
\text { Singh P, Thakur KG, Ringe RP }\end{array}$ & Positiva \\
\hline $\begin{array}{l}\text { Effective in vitro inactivation of SARS- } \\
\text { CoV-2 by commercially available mouthwashes. } \\
\text { (2021) }\end{array}$ & $\begin{array}{c}\text { Davies K, Buczkowski H, Welch SR, Green N, } \\
\text { Mawer D, Woodford N, Roberts ADG, Nixon } \\
\text { PJ, Seymour DW, Killip MJ }\end{array}$ & Negativa \\
\hline $\begin{array}{c}\text { Evaluation of the effects of } \\
\text { chlorhexidine and several flavonoids as antiviral } \\
\text { purposes on SARS-CoV-2 main } \\
\text { protease: molecular docking, molecular dynamics } \\
\text { simulation studies. (2021) }\end{array}$ & Tatar G, Salmanli M, Dogru Y, Tuzuner T & Positiva \\
\hline $\begin{array}{l}\text { Virucidal activity of oral } \\
\text { care products against SARS-CoV-2 in vitro. (2021) }\end{array}$ & $\begin{array}{c}\text { Komine A, Yamaguchi E, Okamoto N, } \\
\text { Yamamoto } \mathrm{K}\end{array}$ & Positiva \\
\hline
\end{tabular}




\begin{tabular}{|c|c|c|}
\hline $\begin{array}{c}\text { Inactivation of } \\
\text { SARS-CoV-2 through Treatment with the Mouth } \\
\text { Rinsing Solutions } \\
\text { ViruProX }{ }^{\circledR} \text { and Bacter } X^{\circledR} \text { Pro. Microorganisms. } \\
\text { (2021) }\end{array}$ & $\begin{array}{c}\text { Koch-Heier J, Hoffmann H, Schindler M, Lussi } \\
\text { A, Planz O }\end{array}$ & Positiva \\
\hline $\begin{array}{l}\text { Comparison of the in-vitro } \\
\text { efficacy of different mouthwash solutions targeting } \\
\text { SARS-CoV-2 based on the } \\
\text { European Standard EN 14476. (2021) }\end{array}$ & $\begin{array}{c}\text { Steinhauer K, Meister TL, Todt D, Krawczyk A, } \\
\text { Paßvogel L, Becker B, Paulmann D, Bischoff } \\
\text { B, Pfaender S, Brill FHH, Steinmann E }\end{array}$ & Negativa \\
\hline
\end{tabular}

Fonte: Nunes, PS; Nunes, CEB. 2021.

em março de 2020 trouxe muitas perguntas e dúvidas em toda a área da saúde e a necessidade de muitas pesquisas e novos estudos para estabelecer, por meio de evidências científicas, quais os melhores e mais eficazes tratamentos e substâncias podem ajudar nesse combate, muitas vezes cruel e solitário.

A saliva contém uma alta carga viral no COVID-19 com até $1,2 \times 108$ copias infecciosas / $\mathrm{ml}$ quando analisada a saliva de pacientes no momento do ingresso no hospital $^{15}$.

O SARS-CoV-2 já foi encontrado em vários tecidos do corpo humano, mas é nas células do pulmão (pneumócitos) e do intestino (enterócitos) que se encontra a maior quantidade de vírus. A possibilidade de transmissão do vírus por aerossóis, tornou-se mais robusta após a identificação de que o ACE2, um receptor de superfície celular necessário para a entrada do vírus na célula humana, também está presente nas células da mucosa oral e que o vírus é encontrado na saliva, apesar de apresentar maior carga viral na orofaringe ${ }^{16}$.

Em um estudo em que os autores investigaram a presença de SARS-COV-2 no tecido periodontal, realizando biópsia post-mortem de vídeo-endoscopia minimamente invasiva em sete casos fatais de COVID-19, usando um sistema de endoscópio regular de vídeo associado a um smartphone para localizar tecido periodontal e, analisando as amostras por RT-PCR, para identificação do RNA SARS-COV-2 e análise histopatológica, encontraram tecido periodontal positivo para SARS-COV-2 (RT-PCR) em cinco casos, demonstrando a presença de SARS-COV -2 em tecido periodontal em pacientes $\mathrm{CO}$ VID-19 positivos ${ }^{17}$.

Desta forma, tanto o biofilme dentário quanto o SARS-CoV-2 podem compartilhar um inimigo comum: enxaguatórios bucais. Amplamente utilizado para auxiliar no controle de doenças como cárie e gengivite, é possível supor que essas soluções desinfetantes possam reduzir temporariamente a contagem de vírus dentro da cavidade oral. Portanto, lavar a boca dos pacientes antes das sessões de atendimento odontológico também pode reduzir a chance de contaminação dentro dos consultórios. Essas suposições levam a uma questão principal: qual solução antisséptica oral deve ser usada ${ }^{18}$

A clorexidina já é amplamente utilizada em hospitais para antissepsia de superfícies cutâneas e mucosas na antissepsia das mãos e antebraços de cirurgiões e assepsia e antissepsia de pacientes cirúrgicos ou pacientes submetidos a procedimentos invasivos e minimamente invasivos, como punções periféricas, colocação de cateteres, tubos vesicais, drenos, cateteres venosos centrais e traqueostomias, todos procedimentos comuns em pacientes internados em unidades de terapia intensiva (UTI) ${ }^{3}$.

Os estudos sobre a redução de carga viral com uso de substâncias como clorexidina, ainda que escassos e com pouco tempo de acompanhamento, começam a fornecer evidências de efetividade. Dos 27 estudos incluídos nesta revisão integrativa, 15 encontraram resultados positivos e recomendam o uso da clorexidina para esse fim, sendo que 6 não observaram correlação positiva para a redução de carga viral e 6 obtiveram resultados inconclusivos.

De acordo com o Conselho Federal de
Odontologia do Brasil (CFO), o melhor é continuar usando as substâncias estabelecidas para reduzir o biofilme e as bactérias presentes na boca. Os mais estudados e que mostraram os melhores resultados a este respeito são digluconato de clorexidina $0,12 \%$ e cloreto de cetilpiridinio $0,05 \%{ }^{19}$.

$\mathrm{O}$ aquecimento, irradiação de luz ultravioleta e produtos químicos podem ser usados para inativar o SARS-CoV-2, mas não há evidências suficientes para apoiar uma medida em relação a outras na prática clínica $^{20}$.

Já foi observado que outras substâncias, tais como formulações Listerine Advanced Defense Sensitive e Total Care e por enxaguatórios bucais comerciais contendo 0,010,02\% de ácido hipocloroso ou 0,58\% de iodo povidona in vitrotestes usando TCF, foram eficazes contra o SARS-COV-2, deixando essas opções como superiores a enxaguatórios bucais com peróxido de hidrogênio ou gluconato de clorexidina, para redução da carga viral de SARS-CoV-2 ${ }^{21}$.

Há ainda uma importância econômica com o uso de clorexidina, que traz um custo-efetividade benéfico para pacientes com COVID-19 em relação a pneuminia ${ }^{22}$.

Outra revisão sistemática buscou mostrar se essa substância seria eficiente na desinfecção de superfícies, mas concluiu-se também que não é eficiente ${ }^{23}$.

\section{CONCLUSÃO}

Com base nos estudos incluídos e seus resultados, pode-se observar que mais pesquisas precisam ser realizadas e certas condições devem estar presentes, como tempo e outros conhecimentos sobre o vírus e suas 


\section{artigo}

variantes, são necessários para que mais evidências sejam encontradas e possam, de forma conclusiva, determinar qual a melhor li- nha de ação com a finalidade de, reduzindo a carga viral, possa trazer benefícios para o tratamento e recuperação de pacientes infectados com o SARS-Cov-2, porém, já existem bons indícios de que a clorexidina possa ser eficiente na redução da carga viral nos casos de COVID-19.

\section{REFERÊNCIAS}

1.Moreira, A., Pinheiro, L. OMS declara pandemia do coronavirus. Disponivel em: http://g1.globo.com. Acesso em 18 de marzo de 2021.

2.Meng, L. et al. Emerging and future challenges for dental and oral medicine. J Dent Res, 2020. 481-7.

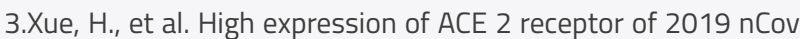
on the epitellial cells of oral mucosa. Int J Oral Sci, 2020,12-8.

4.Welch D, et al. Far-UVC light: uma nova ferramenta para controlar a propagação de doenças microbianas mediadas pelo ar. Sci Rep. 2018; 8: 2752.

5.Doremalen $\mathrm{N}$, et al. Estabilidade de aerossol e superfície do SARS-CoV-2 em comparação com o SARS-CoV-1. N Engl J Med. 2020.

6.Kluk, E. et al. Uma abordagem sobre a clorexidina: ação antimicrobiana e modos de aplicação. Revista Gestão \& Saúde, v. 14, n. 1, p. $07-13,2016$.

7.Denton, G. Chlorhexidine: in disinfection, Sterilization and Preservation. (5 ed.). Philadelphia: Lippincot Williams \& Wilkins. 2001.

8.Zanatta, F., rosing, C. Clorexidina: mecanismos de ação e evidências atuais de sua eficácia no contexto do biofilme supragengival. Scientific-A, 2007, 1(2), 35-43.

9.Fejerskov, O., Kidd, E. Cárie Dentária: a doença e seu tratamento clínico (2 ed.). 2011. São Paulo: Santos.

10.Imran E, et al. Dental Practitioners' Knowledge, Attitude and Practices for Mouthwash Use Amidst the COVID-19 Pandemic. Risk Manag Healthc Policy. 2021 Feb 15;14:605-618.

11. da Silva CC, Ramalho Barbosa da Silva D, Nunes Teixeira G, Figuerêdo Sabino de Lima V, da Silva Ribeiro W. Desafios da prevenção do novo coronavírus (COVID-19) na prática odontológica. SaudColetiv (Barueri) [Internet]. $21^{\circ}$ de outubro de 2020 [citado $30^{\circ}$ de agosto de 2021];10(57):3597-606. Disponível em: http:// www.revistas.mpmcomunicacao.com.br/index.php/saudecoletiva/article/view/950

12.Souza M, et al. Revisão integrativa: o que é e como fazer Integrative review: what is it? How to do it? einstein. 2010; 8(1 Pt 1):102-6.

13.Guimarães, C. Comunicação Científica • Rev. Col. Bras. Cir. 34 (3) . Jun 2007.

14. Ursi ES. Prevenção de lesões de pele no perioperatório: revisão integrativa da literatura. [dissertação]. Ribeirão Preto: Universidade de São Paulo, Escola de

Enfermagem de Ribeirão Preto; 2005.

15.To, K. et al. Consistent detection of 2019 novel. Clinical infec- tious diseases : an official publication of the Infectious. 2020.

16.Matuck, B., et al. Los tejidos periodontales son objetivos de Sars-Cov-2: un estudio post-mortem. Revista de Microbiologia Oral. 2021.

17.Filho JMP, et al. Coronavirus pandemic: is $\mathrm{H} 2 \mathrm{O} 2$ mouthwash going to overcome the chlorhexidine in dental practices? J Stomatol Oral Maxillofac Surg. 2020 Nov 21:S24687855(20)30267-6.

18.Chiewchalermsri C, et al. Chlorhexidine Allergy: Current Challenges and Future Prospects. J Asthma Allergy. 2020; 13: 127133.

19.Franco, A., et al. Atendimento odontológico em UTI's na presença de COVID-19. Interamerican Journal of Medicine and Health, 3, 2020.

20. Kwok CS, Dashti M, Tafuro J, Nasiri M, Muntean EA, Wong N, Kemp T, Hills G, Mallen CD. Methods to disinfect and decontaminate SARS-CoV-2: a systematic review of in vitro studies. Ther Adv Infect Dis. 2021 Mar 16;8:2049936121998548.

21.Davies $K$, et al. Effective in vitro inactivation of SARS-CoV-2 by commercially available mouthwashes. J Gen Virol. 2021 Apr;102(4):001578.

22. Kachapila M, et al. NIHR Global Health Research Unit on Global Surgery; ASOS Investigators; STARSurg Collaborative. Preliminary model assessing the cost-effectiveness of preoperative chlorhexidine mouthwash at reducing postoperative pneumonia among abdominal surgery patients in South Africa. PLoS One. 2021 Aug

\section{2;16(8):e0254698.}

23.Ortega, K. et al. (2020). COVID-19: Qual a efetividade do bochecho pré-procedimento? Rev Assoc Paul Cir Dent, 74-5. 